\title{
CONSEQUENCES OF THE PSEUDO ORBITS TRACING PROPERTY AND EXPANSIVENESS
}

\author{
JERZY OMBACH \\ (Received 17 April 1985; revised 15 December 1985) \\ Communicated by J. F. Price
}

\begin{abstract}
Let $f$ be an expansive homeomorphism with the pseudo orbits tracing property on a compact metric space. There are stable and unstable "manifolds" with similar properties as in the hyperbolic case, and similar behavior near periodic points is observed. $\overline{\operatorname{Per}(f)}=\Omega(f)=C R(f)$. Mappings $\Omega$ and $C R$ are continuous at $f$.
\end{abstract}

1980 Mathematics subject classification (Amer. Math. Soc.): primary 58 F 15; secondary 54 H 20.

\section{Introduction}

This paper is a further attempt to approach some problems of smooth dynamical systems theory from a non-differential point of view. It follows paper [9] where P. Walters emphasized concepts of POTP (pseudo orbits tracing property) and EXPS (expansiveness) proving they are very close to the concept of topological stability. POTP together with EXPS imply topological stability. Topological stability implies POTP. In paper [6] M. Hurley proved that topological stability implies something like Axiom A: he proved the existence of the spectral decomposition of the chain recurrent set $C R(f)$. We try to show here further relations POTP, EXPS and hyperbolicity. As is known, hyperbolicity implies POTP and EXPS; see for example [1], [7], and [9] which provides a good discussion.

\footnotetext{
This research was carried out while the author was a visiting associate professor at Washington State University, 1984-85.

c. 1987 Australian Mathematical Society $0263-6115 / 87 \$ A 2.00+0.00$
} 
Theorems 1 to 4 in this paper show that POTP together with EXPS imply results that are similar to results following from a hypothesis of hyperbolicity. There is a continuous family of stable and unstable "manifolds", a similar situation to the Stable Manifold Theorem ([1], [3], [7]). The situation described by Figure 1 is excluded. There are only three types of periodic points, generally speaking: "sink", "source", and "saddle". For $f \in$ PTOP $\cap$ EXPS, $\overline{\operatorname{Per}(f)}=\overline{\alpha_{f}}$ $=\bar{\omega}_{f}=\Omega(f)=C R(f)$ and mappings $\alpha, \omega, \Omega, C R$ are continuous at $f$ as Theorem 5 shows. In order to emphasize the non-differential character of the results, we work on a compact metric space: only Theorem 5 requires the space to be a manifold. This for example means that the results are valid for any subshift of finite type because it is both POTP and EXPS; see [9].

I am indebted to the referee for his/her suggestions; especially for pointing out to me the facts stated in Proposition 3.

\section{Stable and unstable "manifolds"}

Let $X$ be a compact metric space with some distance $d, f: X \rightarrow X$ a homeomorphism.

A sequence $\left\{x_{n}\right\}_{n \in Z}$ is a $\delta$-pseudo orbit, if for any $n \in Z$,

$$
d\left(f x_{n}, x_{n+1}\right) \leqslant \delta .
$$

A sequence $\left\{x_{n}\right\}_{n \in Z}$ is $\varepsilon$-traced, if there is a point $x$ such that for any $n \in Z$,

$$
d\left(f^{n} x, x_{n}\right) \leqslant \varepsilon .
$$

The homeomorphism $f$ has the pseudo orbits tracing property ( $f \in \mathrm{POTP}$ ), if for any $\varepsilon>0$ there is $\delta>0$ such that any $\delta$-pseudo orbit is $\varepsilon$-traced.

The homeomorphism $f$ is expansive ( $f \in$ EXPS), if there is a number $e(f)>0$ such that

$$
d\left(f^{n} x, f^{n} y\right) \leqslant e(f) \text { for all } n \in Z \text { implies } x=y .
$$

It is easy to see the following is true.

LeMMA 1. Let $n$ be an integer, $n \neq 0$. Then

$$
\begin{aligned}
& f \in P O T P \text { if and only if } f^{n} \in P O T P . \\
& f \in E X P S \text { if and only if } f^{n} \in E X P S .
\end{aligned}
$$

For $N>0$ denote $V_{N}=\left\{(x, y) \in X \times X: d\left(f^{n} x, f^{n} y\right) \leqslant e(f)\right.$ for $\left.|n| \leqslant N\right\}$, where $e(f)$ is an expansive constant of $f$. For $\varepsilon>0$ denote $B_{\varepsilon}=\{(x, y)$ : $d(x, y) \leqslant \varepsilon\}$ and $B(x, \varepsilon)=\{y \in X: d(x, y) \leqslant \varepsilon\}$. 
Lemma 2. Let $f \in E X P S$. Then, for every $\varepsilon>0$ there is a natural $N$ such that $V_{N} \subset B_{\varepsilon}$; for every natural $N$ there is $\varepsilon>0$ such that $B_{\varepsilon} \subset V_{N}$.

This is in fact Lemma 2 from [9].

Define the following sets

$$
\begin{aligned}
& W_{\varepsilon}^{s}(x)=\left\{y: d\left(f^{n} x, f^{n} y\right) \leqslant \varepsilon \text { for all } n \geqslant 0\right\}, \\
& W_{\varepsilon}^{u}(x)=\left\{y: d\left(f^{n} x, f^{n} y\right) \leqslant \varepsilon \text { for all } n \leqslant 0\right\}, \\
& W^{s}(x)=\left\{y: d\left(f^{n} x, f^{n} y\right) \rightarrow 0 \text { as } n \rightarrow \infty\right\}, \\
& W^{u}(x)=\left\{y: d\left(f^{n} x, f^{n} y\right) \rightarrow 0 \text { as } n \rightarrow-\infty\right\} .
\end{aligned}
$$

THEOREM 1. Let $f \in P O T P \cap E X P S$. Then there is a number $\varepsilon_{0}>0$ such that for every $\varepsilon \leqslant \varepsilon_{0}$ there is $\delta>0$ and a continuous function $a: B_{\delta} \rightarrow X$ such that

(A) for every $(x, y) \in X \times X, W_{\varepsilon}^{s}(x) \cap W_{\varepsilon}^{u}(y)$ contains at most one point; for $(x, y) \in B_{\delta}, W_{\varepsilon}^{s}(x) \cap W_{\varepsilon}^{u}(y)=\{a(x, y)\}$;

(B) for every $x \in X$

$$
\begin{aligned}
& W_{\varepsilon}^{s}(x) \cap B(x, \delta)=\{y: y=a(x, y), d(x, y) \leqslant \delta\}, \\
& W_{\epsilon}^{u}(x) \cap B(x, \delta)=\{y: y=a(y, x), d(x, y) \leqslant \delta\} ;
\end{aligned}
$$

(C)

$$
\begin{aligned}
& W_{\varepsilon}^{s}(x) \subset W^{s}(x), \\
& W_{\varepsilon}^{u}(x) \subset W^{u}(x) ;
\end{aligned}
$$

(D)

$$
\begin{aligned}
& W^{\prime}(x)=\bigcup_{n=0}^{\infty} f^{-n}\left(W_{\varepsilon}^{s}\left(f^{n} x\right)\right), \\
& W^{u}(x)=\bigcup_{n=0}^{\infty} f^{n}\left(W_{\varepsilon}^{u}\left(f^{-n} x\right)\right) .
\end{aligned}
$$

Proof. Fix $\varepsilon_{0} \leqslant e(f) / 3$ and take $\varepsilon \leqslant \varepsilon_{0}$. Take $\delta_{1}$ such that $d(x, y) \leqslant \delta_{1}$ implies $d(f x, f y) \leqslant \varepsilon, \delta_{1} \leqslant \varepsilon$. Fix $\delta>0$ such that every $\delta$-pseudo orbit is $\delta_{1}$-traced. Fix $(x, y) \in B_{\delta}$. Now, consider a sequence $\left\{x_{n}\right\}$ defined as

$$
x_{n}= \begin{cases}f^{n} y & \text { for } n<0, \\ f^{n} x & \text { for } n \geqslant 0,\end{cases}
$$

which is a $\delta$-pseudo orbit. It is $\delta_{1}$-traced by some point $a \in X$. There is only one such point. (Suppose $a$ and $a^{\prime}$ are two such points. Then for every $n, d\left(f^{n} a, x_{n}\right)$ $\leqslant \delta_{1}, d\left(f^{n} a^{\prime}, x_{n}\right) \leqslant \delta_{1}$, hence $d\left(f^{n} a, f^{n} a^{\prime}\right) \leqslant 2 \delta_{1} \leqslant 2 \varepsilon \leqslant e(f)$. By expansivenes $a=a^{\prime}$.) So we have defined the mapping $B_{\delta} \ni(x, y) \rightarrow a=a(x, y) \in X$. 
We have for $(x, y) \in B_{\delta}$

$$
\begin{array}{ll}
d\left(f^{n} x, f^{n} a(x, y)\right) \leqslant \delta_{1} \leqslant \varepsilon & \text { for } n \geqslant 0, \\
d\left(f^{n} y, f^{n} a(x, y)\right) \leqslant \delta_{1} \leqslant \varepsilon & \text { for } n<0 .
\end{array}
$$

In particular $d\left(f^{-1} y, f^{-1} a(x, y)\right) \leqslant \delta_{1}$ and by choice of $\delta_{1}, d(y, a(x, y) \leqslant \varepsilon$. Hence

$$
d\left(f^{n} y, f^{n} a(x, y)\right) \leqslant \varepsilon \text { for } n \leqslant 0 .
$$

(1) and (2) imply that $a(x, y) \in W_{\varepsilon}^{s}(x) \cap W_{\varepsilon}^{u}(y)$. Moreover, this intersection cannot contain two distinct points. If $b, c \in W_{\varepsilon}^{s}(x) \cap W_{\varepsilon}^{u}(y)$, then for every $n \geqslant 0, d\left(f^{n} b, f^{n} c\right) \leqslant d\left(f^{n} b, f^{n} x\right)+d\left(f^{n} x, f^{n} c\right) \leqslant 2 \varepsilon \leqslant e(f)$ and similarly for $n \leqslant 0, d\left(f^{n} b, f^{n} c\right) \leqslant d\left(f^{n} b, f^{n} y\right)+d\left(f^{n} y, f^{n} c\right) \leqslant 2 \varepsilon \leqslant e(f)$. By expansiveness $b=c$, and hence (A) is proved.

To prove the continuity of the mapping $a$ we use Lemma 2. Let $(x, y)$, $\left(x^{\prime}, y^{\prime}\right) \in B_{\delta}$. For $n \geqslant 0$ we have from (1)

$$
\begin{aligned}
d\left(f^{n} a(x, y),\right. & \left.f^{n} a\left(x^{\prime}, y^{\prime}\right)\right) \\
& \leqslant d\left(f^{n} a(x, y), f^{n} x\right)+d\left(f^{n} x, f^{n} x^{\prime}\right)+d\left(f^{n} x^{\prime}, f^{n} a\left(x^{\prime}, y^{\prime}\right)\right) \\
& \leqslant 2 \varepsilon+d\left(f^{n} x, f^{n} x^{\prime}\right) .
\end{aligned}
$$

For $n \leqslant 0$ we have from (2)

$$
\begin{aligned}
d\left(f^{n} a(x, y),\right. & \left.f^{n} a\left(x^{\prime}, y^{\prime}\right)\right) \\
& \leqslant d\left(f^{n} a(x, y), f^{n} y\right)+d\left(f^{n} y, f^{n} y^{\prime}\right)+d\left(f^{n} y^{\prime}, f^{n} a\left(x^{\prime}, y^{\prime}\right)\right) \\
& \leqslant 2 \varepsilon+d\left(f^{n} y, f^{n} y^{\prime}\right) .
\end{aligned}
$$

Now fix some $\mu>0$. Lemma 2 produces $N$ with $V_{N} \subset B_{\mu}$. Continuity of $f^{k}$ with $|k| \leqslant N$ implies that there is a $\lambda$ such that for $\left(x, x^{\prime}\right),\left(y, y^{\prime}\right) \in B_{\lambda}$ we have $d\left(f^{n} x, f^{n} x^{\prime}\right) \leqslant \varepsilon$ for $0 \leqslant n \leqslant N$ and $d\left(f^{n} y, f^{n} y^{\prime}\right) \leqslant \varepsilon$ for $-N \leqslant n \leqslant 0$. So, for such $x, x^{\prime}, y, y^{\prime}$ we get

$$
d\left(f^{n} a(x, y), f^{n} a\left(x^{\prime}, y^{\prime}\right)\right) \leqslant 3 \varepsilon \leqslant e(f) \text { for }|n| \leqslant N,
$$

which means that $\left(a(x, y), a\left(x^{\prime}, y^{\prime}\right)\right) \in V_{N} \subset B_{\mu}$, so the mapping is continuous.

To prove (B) let us note first, $y=a(x, y) \in W_{\varepsilon}^{s}(x)$ by (1) and second, for $y \in W_{\varepsilon}^{s}(x)$ we see $y \in W_{\varepsilon}^{s}(x) \cap W_{\varepsilon}^{u}(y)$. Since $(x, y) \in B_{\delta}, y=a(x, y)$. The proof of the remaining case is the same.

Now we prove (C). Let $y \in W_{\varepsilon}^{s}(x)$. Given $\mu$ there is $N$ by Lemma 2 such that $V_{N} \subset B_{\mu}$. By the definition of $W_{\varepsilon}^{s}(x)$ we see that for $n \geqslant N,\left(f^{n} x, f^{n} y\right) \in V_{N} \subset$ $B_{\mu}$, but this meanse that $d\left(f^{n} x, f^{n} y\right) \rightarrow 0$ as $n \rightarrow \infty$. Hence $y \in W^{s}(x)$. Similarly $W_{\varepsilon}^{u}(x) \subset W^{u}(x)$.

Proving (D) is now trivial, the proof of Theorem 1 is completed. 


\section{Sinks, sources, saddles}

In this section we display three possible types of behaviour of orbits near a point from the space $X$. We define a sink, a source, a saddle in a case the point is periodic and as we will see in Theorems 2 to 4 such names are justified by excellent similarity to the hyperbolic case. Propositions 1 to 5 contain the main steps for proofs of these theorems.

We assurne in the sequel that $f \in \operatorname{POTP} \cap$ EXPS, $x \in X$, and $0 \leqslant \varepsilon \leqslant \varepsilon_{0}$, where $\varepsilon_{0}$ is given in Theorem 1 .

Define the positive and negative limit set

$$
\begin{aligned}
& \omega_{f}(x)=\omega(x)=\left\{y: f^{k_{n}} x \rightarrow y, \text { for some } k_{n} \rightarrow \infty\right\}, \\
& \alpha_{f}(x)=\alpha(x)=\left\{y: f^{k_{n}} x \rightarrow y, \text { for some } k_{n} \rightarrow-\infty\right\} .
\end{aligned}
$$

First we show that the situation illustrated by Figure 1 is excluded. A related problem has been considered in a different context in $[4,6.16]$.

Proposition 1. If int $W^{s}(x) \neq \varnothing$, then $x \in \operatorname{int} W^{s}(x)$.

Proof. Take $y \in X$ and $\varepsilon_{1}>0$ such that $y \in B\left(y, \varepsilon_{1}\right) \subset W^{s}(x), \varepsilon_{1} \leqslant \varepsilon_{0} / 2$, where $\varepsilon_{0}$ is given in Theorem 1. Choose some $\delta$ corresponding to $\varepsilon_{1}$ by POTP. Continuity of $f$ provides a $\lambda>0$ such that

$$
d(u, v) \leqslant \lambda \text { implies } d(f u, f v) \leqslant \delta .
$$

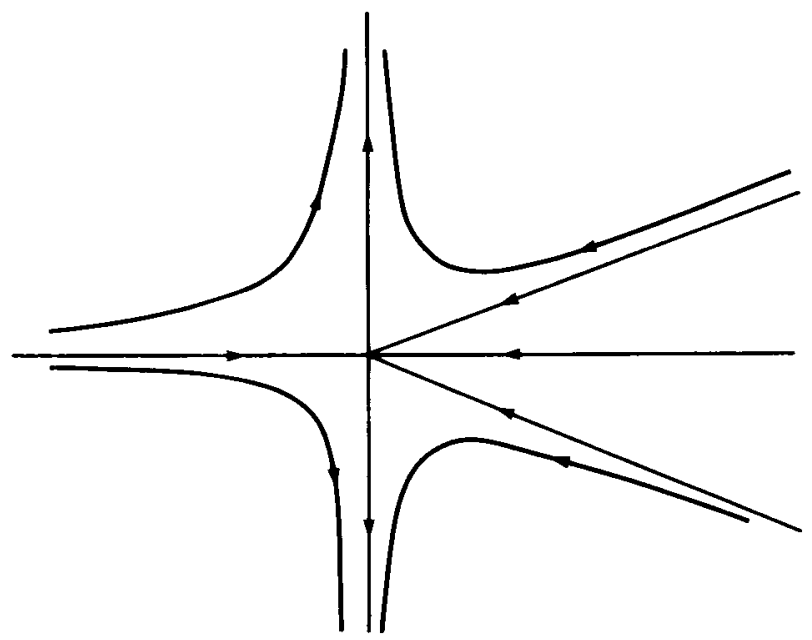

Figure 1 
There is $N>0$ such that

$$
d\left(f^{N} x, f^{N} y\right) \leqslant \frac{1}{2} \lambda
$$

because $y \in W^{s}(x)$. By continuity of $f^{N}$ there is a neighborhood $U$ of $x$ such that for each $x^{\prime} \in U$

$$
d\left(f^{N} x^{\prime}, f^{N} x\right) \leqslant \frac{1}{2} \lambda
$$

It is enough to show that $U \subset W^{s}(x)$. Let $x^{\prime} \in U$. By (4) and (5) $d\left(f^{N} x^{\prime}, f^{N} y\right)$ $\leqslant \lambda$ and by (3) the sequence defined by

$$
x_{n}= \begin{cases}f^{n} y & \text { for } n \leqslant N, \\ f^{n} x^{\prime} & \text { for } n>N,\end{cases}
$$

is a $\delta$-pseudo orbit. It is $\varepsilon_{1}$-traced by some point $y^{\prime}$. This implies in particular

$$
\begin{gathered}
d\left(y^{\prime}, y\right) \leqslant \varepsilon_{1}, \\
d\left(f^{n} y^{\prime}, f^{n} x^{\prime}\right) \leqslant \frac{1}{2} \varepsilon_{0} \quad \text { for } n>N .
\end{gathered}
$$

Now (6) implies that $y^{\prime} \in W^{s}(x)$, and hence there is $N^{\prime}$ such that for $n \geqslant N^{\prime}$, $d\left(f^{n} y^{\prime}, f^{n} x\right) \leqslant \varepsilon_{0} / 2$ which together with (7) imply that for large $n, f^{n} x^{\prime} \in$ $W_{\varepsilon_{0}}^{s}\left(f^{n} x\right)$. Hence by Theorem 1(D) $x^{\prime} \in W^{s}(x)$ and the proof is completed.

Proposition 2. If $x \in \operatorname{int} W^{s}(x)$, then $x \in \operatorname{int} W_{\varepsilon}^{s}(x)$.

Proof. Let $x \in \operatorname{int} W^{s}(x)$. It is enough to have a number $N$ and a neighborhood $U$ of $x$ such that for $n \geqslant N$ and $y \in U, d\left(f^{n} x, f^{n} y\right) \leqslant \varepsilon$. Then, continuity of $f^{n}$ for $0 \leqslant n \leqslant N$ will finish the proof. Asssume this is not true, that is,

$$
\begin{aligned}
& \text { for any number } N \text { and any neighborhood } U \text { of } x \text { there } \\
& \text { is } n>N \text { and } y \in U \text { such that } d\left(f^{n} x, f^{n} y\right)>\varepsilon \text {. }
\end{aligned}
$$

Let $B\left(x, \varepsilon_{1}\right) \subset W^{s}(x), \varepsilon_{1}>0$ and let $\delta>0$ be such a number that any $\delta$-pseudo orbit is $\min \left(\varepsilon / 2, \varepsilon_{1}\right)$-traced. Let $\eta>0$ be chosen, depending on $\delta$, by continuity of $f$.

Lemma 3. There is a $\delta$-pseudo orbit $\left\{z_{n}\right\}$, there are numbers $l_{i}, n_{i}, i=1,2,3, \ldots$, $0=l_{1}<n_{2}<l_{2}<n_{3}<\ldots$, such that

$$
\begin{gathered}
d\left(z_{n_{t}}, f^{n_{i}} x\right)>\varepsilon_{1}, \\
z_{l_{i}}=f^{l} x
\end{gathered}
$$

for $i=1,2,3, \ldots$ 
Proof. Define $z_{n}=f^{n} x$ for $n \leqslant 0$ and put $l_{1}=0$. Assume that we have already defined numbers $n_{i}, l_{i}$ for $i \leqslant j$ and points $z_{n}$ for $n \leqslant l_{j}$, such that (9) and (10) hold and $d\left(f z_{n}, z_{n+1}\right) \leqslant \delta$ for $n<l_{j}$. Since $x \in$ int $W^{s}(x), f$ is continuous and (8) holds, we can find a point $y \in W^{s}(x)$, a number $n_{j+1}>l_{j}$ such that $d\left(f^{\prime} y, z_{j}\right)=d\left(f^{l_{j}} y, f^{l_{j}} x\right)<\eta$ and $d\left(f^{n_{j+1}} y, f^{n_{j+1}} x\right)>\varepsilon$. Since $y \in W^{s}(x)$ there is a number $l_{j+1}>n_{j+1}$ such that $d\left(f^{1_{j+1}-1} y, f^{l_{j+1}-1} x\right)<\eta$.

Put $z_{n}=f^{n} y$ for $l_{j}<n<l_{j+1}$ and $z_{l_{j+1}}=f^{l_{j+1} x}$. The choice of $\eta$ implies $d\left(f z_{n}, z_{n+1}\right)<\delta$ for $n<l_{j+1}$ and (9), (10) are also satisfied for $j+1$. Thus the lemma is proved.

To finish the proof of Proposition 2 take a point $x^{\prime}$ tracing the pseudo orbit $\left\{z_{n}\right\}$. We get $d\left(f^{n} x^{\prime}, z_{n}\right) \leqslant \min \left(\frac{1}{2} \varepsilon, \varepsilon_{1}\right)$. In particular, $x^{\prime} \in B\left(x, \varepsilon_{1}\right) \subset W^{s}(x)$, that means $d\left(f^{n} x^{\prime}, f^{n} x\right) \rightarrow 0$, as $n \rightarrow \infty$, and $d\left(f^{n_{i}} x^{\prime}, z_{n_{i}}\right) \leqslant \frac{1}{2} \varepsilon$ for infinitely many $n_{i}$. The triangle inequality leads us to a contradiction with (8).

The idea of the next proposition was suggested to me by the referee.

Proposition 3. If $x \in \operatorname{int} W_{\varepsilon / 4}^{s}(x)$, then $\omega(x)$ is a periodic orbit equal to $\left\{y, f y, \ldots, f^{k-1} y\right\}$ and $y \in$ int $W^{s}(y)$.

Proof. Let $B\left(x, \varepsilon_{1}\right) \subset W_{\varepsilon / 4}^{s}(x) \subset W^{s}(x), \varepsilon_{1}>0$. It is known that $\omega(x) \neq \varnothing$ in a compact space and $\omega(y) \subset \omega(x)$ for any $y \in \omega(x)$. To show that $\omega(y)=$ $\omega(x)$ for such a $y$. Let $z \in \omega(x)$ and $\mu>0$. Take $\delta>0$ in such a way that any $\delta$-pseudo orbit is $\min \left(\varepsilon_{1}, \mu / 3\right)$-traced. The $\delta$-pseudo orbit

$$
z_{n}= \begin{cases}f^{n} x & \text { for } n<N, \\ f^{n-N} y & \text { for } n \geqslant N\end{cases}
$$

where $N$ is a number with $d\left(f^{N} x, y\right)<\delta$, is $\mu / 3$-traced by a point $x^{\prime} \in W^{s}(x)$. So we get

$$
\begin{aligned}
d\left(f^{n} x, f^{n \prime}\right) \leqslant \mu / 3 & \text { for large } n, \\
d\left(f^{n} x, z\right) \leqslant \mu / 3 & \text { for infinitely many } n, \text { and } \\
d\left(f^{n-N} y, f^{n} x^{\prime}\right) \leqslant \mu / 3 & \text { for } n \geqslant N .
\end{aligned}
$$

Thus $d\left(f^{n} y, z\right) \leqslant \mu$ for some $n$, and hence $z \in \omega(y)$.

Let any $\alpha$-pseudo orbit be $\min \left(\varepsilon_{1}, \varepsilon / 4\right)$-traced, $\alpha>0$. We show that $B(y, \alpha / 2)$ $\subset W_{\varepsilon}^{s}(y)$ for any $y$ from $\omega(x)$. Let $N$ be such that $d\left(f^{N} x, y\right) \leqslant \alpha / 2$ and let $z \in B(y, \alpha / 2)$. The sequences defined as follows

$$
y_{n}=\left\{\begin{array}{ll}
f^{n} x & \text { for } n<N, \\
f^{n-N_{y}} & \text { for } n \geqslant N,
\end{array} \quad z_{n}= \begin{cases}f^{n} x & \text { for } n<N, \\
f^{n-N} z & \text { for } n \geqslant N,\end{cases}\right.
$$


are both $\alpha$-pseudo orbits. They are $\varepsilon / 4$-traced by points $y^{\prime}$ and $z^{\prime}$ from $W_{\varepsilon / 4}^{s}(x)$. For $n \geqslant 0$ we have

$$
\begin{aligned}
d\left(f^{n} z, f^{n} y\right)= & d\left(z_{n+N}, y_{n+N}\right) \\
\leqslant & d\left(z_{n+N}, f^{n+N} z^{\prime}\right)+d\left(f^{n+N z^{\prime}}, f^{n+N} x\right)+d\left(f^{n+N} x, f^{n+N} y^{\prime}\right) \\
& +d\left(f^{n+N} y^{\prime}, y_{n+N}\right) \leqslant \varepsilon .
\end{aligned}
$$

That means $y \in \operatorname{int} W_{\varepsilon}^{s}(y) \subset \operatorname{int} W^{s}(y)$.

It is known (see [8] and Proposition 6 in this paper) that any neighborhood of a limit point contains a periodic one. Let $p \in W^{s}(y)$ be a periodic point. We claim $p=y$. For $p \neq y$ we would find another periodic point $q \in W^{s}(y), q \neq p$. Hence $W^{s}(p)=W^{s}(y)=W^{s}(q)$, so $d\left(f^{n} p, f^{n} q\right) \rightarrow 0$, which is impossible.

Thus we have $\omega(x)=\omega(y)=\left\{y, f y, \ldots, f^{k-1} y\right\}$, where $k>0$ is a period of $y$.

Proposition 4. $x \in \operatorname{int} W_{\varepsilon}^{s}(x)$ if and only if $W_{\varepsilon}^{u}(x) \cap H=\{x\}$, where $H$ is some neighborhood of $x$.

Proof. Let $x \in \operatorname{int} W_{\varepsilon}^{s}(x)$. Take a neighborhood $H$ of $x$ such that $H \subset W_{\varepsilon}^{s}(x)$ $\cap B(x, \delta)$, where $\delta$ is chosen in terms of $\varepsilon$ as in Theorem 1. For $y \in W_{\varepsilon}^{u}(x) \cap H$ we have, $y \in W_{\varepsilon}^{s}(x) \cap W_{\varepsilon}^{u}(x)=\{a(x, x)\}=\{x\}$.

Let $W_{\varepsilon}^{u}(x) \cap H=\{x\}$, where $H$ is a neighborhood of $x$. Continuity of the mapping $a: B_{\delta} \rightarrow X$ (Theorem 1) provides a neighborhood $U$ of $x$ such that $y \in U$ implies $a(y, x) \in H$. Since $a(y, x) \in W_{\varepsilon}^{u}(x)$, we have $a(y, x)=x$ and $y \in W_{\varepsilon}^{s}(x)$, and hence $U \subset W_{\varepsilon}^{s}(x)$.

Proposition 5. Let $x \in \operatorname{int} W_{\varepsilon}^{s}(x)$ be a periodic point with period $k$ and let $\varepsilon \leqslant e\left(f^{k}\right)$ (see Lemma 1). Then there is a neighborhood $G$ of $x$ such that

$$
\begin{gathered}
f^{k}(G) \subset G, \\
\bigcap_{n=0}^{\infty} f^{n k}(G)=\{x\} .
\end{gathered}
$$

Let us note that conditions (11), (12) mean the periodic orbit $\left\{x, f x, \ldots, f^{k-1} x\right\}$ is an attractor in the sence of Conley ([2], [4]).

Proof. Let $x \in G_{1} \subset \operatorname{int} W_{\varepsilon}^{s}(x), G_{1}$ open. For every $n \geqslant 0, f^{n k}\left(G_{1}\right) \subset B(x, \varepsilon)$. Define $G=\bigcup_{n=0}^{\infty} f^{n k}\left(G_{1}\right)$. Then $G$ is a neighborhood of $x$ and (11) holds true. Furthermore, $G \subset B(x, \varepsilon)$. Let $x^{\prime} \subset \cap_{n=0}^{\infty} f^{n k}(G)$. Then, in particular $x^{\prime} \in G$ and by (11) $x^{\prime} \in f^{n k}(G)$ for any $n<0$. So for any $n \in Z, x^{\prime} \in f^{n k}(B(x, \varepsilon))$ and hence for all $n, d\left(f^{k n} x^{\prime}, f^{k n} x\right)=d\left(f^{k n} x^{\prime}, x\right) \leqslant \varepsilon \leqslant e\left(f^{k}\right)$. So $x^{\prime}=x$ and (12) is proved.

The following theorem summarizes and completes all previous results. 
THEOREM 2. The following conditions are equivalent for a point $x \in X, 0<\varepsilon \leqslant \varepsilon_{0}$, $y \in \omega(x)$.

(AS) int $W^{s}(x) \neq \varnothing$

(BS) $x \in \operatorname{int} W^{s}(x)$,

(CS) $W^{s}(x)$ is open,

(DS) $x \in \operatorname{int} W_{\varepsilon}^{s}(x)$,

(ES) int $W_{\varepsilon}^{s}(x) \neq \varnothing$,

(FS) $W_{\varepsilon}^{u}(x) \cap H=\{x\}$, where $H$ is some neighborhood of $x$,

(GS) $W_{\varepsilon}^{u}(y)=\{y\}$,

(HS) $W^{u}(y)=\{y\}$,

(IS) $\omega(x)=\left\{y, f y, \ldots, f^{k-1} y\right\}$ is a periodic orbit.

There is a neighborhood $G$ of $y$ such that

$$
\begin{gathered}
f^{k}(G) \subset G, \\
\bigcap_{n=0}^{\infty} f^{n k}(G)=\{y\} .
\end{gathered}
$$

Proof. (AS) $\Rightarrow^{\prime}(\mathrm{BS})$ by Proposition 1. To see that (BS) $\Rightarrow(\mathrm{CS})$ consider a point $z \in W^{s}(x)$. Then $W^{s}(z)=W^{s}(x)$ contains $x$ in its interior. By Proposition $1, z \in \operatorname{int} W^{s}(z)=\operatorname{int} W^{s}(x)$, and hence $W_{e}^{s}(x)$ is open. (CS) $\Rightarrow$ (DS) by Proposition 2. (DS) $\Rightarrow$ (ES) is trivial. (ES) $\Rightarrow$ (AS) by Theorem 1(C). (DS) $\Leftrightarrow$ (FS) by Proposition 4. Thus we have proved equivalences among conditions (AS) to (FS).

We prove (AS) $\Rightarrow$ (IS). By Proposition 2, $x \in \operatorname{int} W_{e_{0}}^{s}(x)$. By Proposition 3, $y \in \operatorname{int} W^{s}(y)$ and $y$ is periodic. Once again by Proposition 2, $y \in \operatorname{int} W_{\varepsilon_{1}}^{s}(y)$ for small $\varepsilon_{1}>0$. Proposition 5 completes the proof.

To see (IS) $\Rightarrow$ (HS) take some $z \neq y$. Then for all $n$ large enough, $z \notin f^{n k}(G)$, as (13) implies the sequence $f^{n k}(G)$ decreases. So $f^{-n k} z \notin G$ for such $n$ and $z \notin W^{u}(y)$.

$(\mathrm{HS}) \Rightarrow$ (GS) by Theorem 1(C).

We prove (GS) $\Rightarrow$ (DS). $W_{\varepsilon}^{u}(y)=\{y\}$ implies $W_{\varepsilon / 2}^{u}(y)=\{y\}$ and by Proposition 4, $y \in \operatorname{int} W_{\varepsilon / 2}^{s}(y)$. As $y \in \omega(x)$, then there is a number $N$ such that $f^{N} x \in$ int $W_{\varepsilon / 2}^{s}(y)$. Continuity of $f^{n}, 0 \leqslant n \leqslant N$, provides a neighborhood $U$ of $x$ such that for $z \in U, d\left(f^{n} x, f^{n} z\right) \leqslant \varepsilon$ for $0 \leqslant n \leqslant N$ and $f^{N_{z}} \in W_{\varepsilon / 2}^{s}(y)$. It is clear that $U \subset W_{\varepsilon}^{s}(x)$, so (DS) is true.

The proof of Theorem 2 is complete.

Applying Theorem 2 to the inverse of $f$ we immediately get

THEOREM 3. The following conditions are equivalent for a point $x \in X, 0<\varepsilon<\varepsilon_{0}$, $y \in \alpha(x)$.

(AU) int $W^{u}(x) \neq \varnothing$,

(BU) $x \in \operatorname{int} W^{u}(x)$, 
(CU) $W^{u}(x)$ is open,

(DU) $x \in \operatorname{int} W_{\varepsilon}^{u}(x)$,

(EU) int $W_{\varepsilon}^{u}(x) \neq \varnothing$,

(FU) $W_{\varepsilon}^{s}(x) \cap H=\{x\}$, where $H$ is some neighborhood of $x$,

(GU) $W_{\varepsilon}^{s}(y)=\{y\}$,

(HU) $W^{s}(y)=\{y\}$,

(IU) $\alpha(x)=\left\{y, f y, \ldots, f^{k-1} y\right\}$ is a periodic orbit.

There is a neighborhood $G$ of $y$ such that

$$
f^{-n}(G) \subset G, \quad \bigcap_{n=0}^{\infty} f^{-n k}(G)=\{y\} .
$$

Assume in the sequel that $x$ is a non-isolated point in the space $X$ and $y \in \omega(x)$. Let us note that the situations described in Theorems 2 and 3 exclude each other. To see this, compare conditions (DS) and (FU). There is just one other interesting situation. It is enough to combine the negation of a condition from (AS) to (IS) with the negation of a condition from (AU) to (IU) to get the description of this third situation. Taking advantage of (FS) and (FU), for example, we get

any neighborhood of $x$ contains points

$$
x_{s} \in W_{\varepsilon}^{s}(x) \backslash\{x\}, \quad x_{u} \in W_{\varepsilon}^{u}(x) \backslash\{x\}, \quad x_{s} \neq x_{u} .
$$

Here $x_{s} \neq x_{u}$ since Theorem 1 implies $W_{\varepsilon}^{s}(x) \cap W_{\varepsilon}^{u}(x)=\{x\}$.

LeMma 4. If a point $x$ is periodic, then condition $(\mathrm{Y})$ is equivalent to $(\mathrm{X})$

any neighborhood of $x$ contains points

$$
x_{s} \in W^{s}(x) \backslash\{x\}, \quad x_{u} \in W^{u}(x) \backslash\{x\}, \quad x_{s} \neq x_{u} .
$$

Proof. $(\mathrm{Y}) \Rightarrow(\mathrm{X})$ by Theorem $1(\mathrm{C})$. To see $(\mathrm{X}) \Rightarrow(\mathrm{Y})$, take advantage of $(\mathrm{HS}) \Leftrightarrow(\mathrm{FS})$ and $(\mathrm{HU}) \Leftrightarrow(\mathrm{FU})$ in the case $y=x \in \omega(x)=\alpha(x)$.

Now, it makes sense to call a periodic point a sink, if the situation described in Theorem 2 occurs, a source, if the situation described in Theorem 3 occurs, a saddle, if the situation described in Lemma 4 occurs.

Similarities to the hyperbolic case are clear. Thus we have

THEOREM 4. Any periodic point is either a sink or a source or a saddle.

\section{Continuous dependence of sets describing asymptotic behavior}

For a homomorphism $f: X \rightarrow X$ define the periodic set

$$
\operatorname{Per}(f)=\left\{x \in X: f^{k} x=x, \text { for some } k\right\},
$$


the negative and positive limit set

$$
\alpha_{f}=\bigcup_{x \in X} \alpha_{f}(x), \quad \omega_{f}=\bigcup_{x \in X} \omega_{f}(x),
$$

the non-wandering set

$\omega(f)=\{x \in X$ : for every neighborhood $U$ of $x$ there is

$$
\left.n \neq 0 \text { such that } f^{n}(U) \cap U \neq \varnothing\right\},
$$

the chain recurrent set

$$
C R(f)=\{x \in X \text { : for every } \varepsilon>0 \text { there is an } \varepsilon \text {-chain from } x \text { to itself }\}
$$

where an $\varepsilon$-chain from $x$ to itself is a finite $\varepsilon$-pseudo orbit $\left\{x_{0}, \ldots, x_{n}\right\}$, $x=x_{0}=x_{n}, n>0$. It is known and not hard to prove that $\Omega(f)$ and $C R(f)$ are closed and

$$
\operatorname{Per}(f) \subset \alpha_{f} \cap \omega_{f} \subset \alpha_{f} \cup \omega_{f} \subset \Omega(f) \subset C R(f) .
$$

The following summarizes known results (see [1], [8]).

Proposition 6. Let $f \in P O T P \cap$ EXPS. Then

$$
\overline{\operatorname{Per}(f)}=\overline{\alpha_{f}}=\overline{\omega_{f}}=\Omega(f)=C R(f) .
$$

Proof. We have to show that $C R(f) \subset \overline{\operatorname{Per}(f)}$. Let $x \in C R(f)$ and $\varepsilon \leqslant \frac{1}{2} e(f)$ be fixed. POTP provides a $\delta$ corresponding to this $\varepsilon$. There is a $\delta$-chain $\left\{x_{0}, \ldots, x_{N}\right\}$ from $x$ to itself. The sequence $\left\{x_{n}^{\prime}\right\}, n \in Z$ defined by $x_{n}^{\prime}=x_{i}$ if $n=i(\bmod N)$, is a $\delta$-pseudo orbit. It is $\varepsilon$-traced by some point $y$, but also by $f^{N} y$. The triangle inequality implies for all $n, d\left(f^{n} y, f^{n N} y\right) \leqslant 2 \varepsilon \leqslant e(f)$, and hence $y=f^{N} y$. Thus $y \in \operatorname{Per}(f)$ and $d(y, x)=d\left(y, x_{0}\right) \leqslant \varepsilon$.

Define the metric space $H(X)=\{f: X \rightarrow X, f$ is a homeomorphism $\}$ with the distance $d_{H}(f, g)=\sup \{d(f x, g x), x \in X\}$ and the metric space $K(X)=\{K$ $\subset X: K$ is closed $\}$ with the Hausdorff distance $H(K, L)=\max \left(\max _{x \in K} d(x, L)\right.$, $\left.\max _{y \in L} d(y, L)\right)$.

Let $\alpha, \omega, \Omega, C R$ denote mappings $H(X) \rightarrow K(X)$ sending $f$ to $\bar{\alpha}_{f}, \bar{\omega}_{f}, \Omega(f)$, $C R(f)$, respectively.

THEOREM 5. Let $X$ be a compact manifold. Then the mappings defined above are continuous at points from the set POTP $\cap$ EXPS.

First we recall the concept of upper and lower semicontinuity. Let $T$ be a topological space. A mapping $F: T \rightarrow K(X)$ is upper semi-continuous at $t_{0} \in T$, if for every $\varepsilon>0$ there is a neighborhood of $t_{0}$ such that for $t$ belonging to it we 
have $F(t) \subset U_{\varepsilon}\left(F\left(t_{0}\right)\right)$, and lower semi-continuous at $t_{0} \in T$, if for every $\varepsilon>0$ there is a neighborhood of $t_{0}$ susch that for $t$ belonging to it we have $F\left(t_{0}\right) \subset$ $U_{\varepsilon}(F(t))$, where $U_{\varepsilon}(K)=\{y: d(y, x) \leqslant \varepsilon$, with some $x \in K\}$; see [2], [4] and references therein.

The following lemma is obvious.

LeMMA 5. A mapping $F: T \rightarrow K(X)$ is continuous at $t_{0}$ if and only if $F$ is upper and lower semi-continuous at $t_{0}$.

The following lemma contains the result from [5]; compare also with [2, Section II, 6.2A], where upper semi-continuity of the chain recurrent set $C R(S)=R(S)$ as a mapping of a compact invariant set $S$ has been proved.

LEMMA 6. The mapping $C R: H(X) \rightarrow K(X)$ is upper semi-continuous at every point $f_{0} \in K(X)$.

ProOf. For any $\varepsilon>0$ define $C R_{\varepsilon}(f)=\{x \in X$ : there is an $\varepsilon$-chain from $x$ to itself $\}$. We see that $\bigcap_{\varepsilon>0} C R_{\varepsilon}(f)=C R(f), C R_{\varepsilon_{1}}(f) \subset C R_{\varepsilon_{2}}(f)$ for $\varepsilon_{1} \leqslant \varepsilon_{2}$. Now fix $\varepsilon>0$. 'There is $\mu>0$ such that $C R_{\mu}\left(f_{0}\right) \subset U_{\varepsilon}\left(C R\left(f_{0}\right)\right)$; let $\delta=\mu / 2$. For $f \in H(X)$ with $d_{H}\left(f, f_{0}\right) \leqslant \delta$ we show that $C R(f) \subset C R_{\mu}\left(f_{0}\right) \subset U_{\varepsilon}\left(C R\left(f_{0}\right)\right)$. Let $x \in C R(f)$ and let $\left\{x_{0}, \ldots, x_{N}\right\}$ be a $\delta$-chain with respect to $f$. It is also the $\mu$-chain with respect to $f_{0}$ by the triangle inequality, so the proof is completed.

We will take advantage of the following.

WALTERs' TheOREM [9]. Any homeomorphism $f_{0} \in$ POTP $\cap$ EXPS is topologically stable, that is, there is $\varepsilon_{0}$ susch that for any $\varepsilon \leqslant \varepsilon_{0}$ there is $\delta>0$ such that for any homeomorphism $f$ with $d_{H}\left(f, f_{0}\right) \leqslant \delta$ there is a continuous mappnig $h: X \rightarrow X$ satisfying

$$
\begin{gathered}
d_{H}(i d, h) \leqslant \varepsilon, \\
f_{0} \circ h=h \circ f .
\end{gathered}
$$

In case the space $X$ is a manifold the mapping $h$ is "onto".

LEMMA 7. If $X$ is a compact manifold and $f_{0} \in P O T P \cap E X P S$, then the mappings $\alpha$ and $\omega$ are lower semi-continuous at $f_{0}$.

Proof. For given $\varepsilon>0$ take $\delta>0$ as in Walters' Theorem and fix $f \in H(X)$ with $d_{H}\left(f, f_{0}\right) \leqslant \delta$. There is a mapping $h: X \rightarrow X$, which is onto $X$ and $(17),(18)$ hold. For any $x \in X$ there is $x^{\prime} \in X$ such that $x=h\left(x^{\prime}\right)$ and since (18) implies 
for any $n, f_{0}^{n} \circ h=h \circ f^{n}$, we have $f_{0}^{n} x=h f^{n} x^{\prime}$. From (17) we see that

$$
d\left(f_{0}^{n} x, f^{n} x^{\prime}\right)=d\left(h f^{n} x^{\prime}, f^{n} x^{\prime}\right) \leqslant \varepsilon .
$$

Thus $\omega_{f_{0}}(x) \subset U_{\varepsilon}\left(\omega_{f}\left(x^{\prime}\right)\right) \subset U_{\varepsilon} \overline{\left(\omega_{f}\right)}$, and at last $\overline{\omega_{f_{0}}} \subset U_{\varepsilon} \overline{\left(\omega_{f}\right)}$. The proof for the mapping $\alpha$ is the same.

Proof of Theorem 5. Let $f_{0} \in$ POTP $\cap$ EXPS, $\varepsilon>0$. Let $d_{H}\left(f, f_{0}\right) \leqslant \delta_{1}$ where $\delta_{1}$ is chosen to $\varepsilon$ as in Lemma 6. Thus $C R(f) \subset U_{\varepsilon}\left(C R\left(f_{0}\right)\right)$. By (15) and (16)

$$
\begin{aligned}
\overline{\alpha_{f}} \cup \overline{\omega_{f}} \subset \Omega(f) \subset C R(f) \subset U_{\varepsilon}\left(C R\left(f_{0}\right)\right) \\
=U_{\varepsilon}\left(\Omega\left(f_{0}\right)\right)=U_{\varepsilon}\left(\overline{\alpha_{f_{0}}}\right)=U_{\varepsilon}\left(\overline{\omega_{f_{0}}}\right)
\end{aligned}
$$

which means upper semi-continuity at $f_{0}$ of all considered mappings.

Let $d_{H}\left(f, f_{0}\right) \leqslant \delta_{2}$ where $\delta_{2}$ is chosen in terms of $\varepsilon$ as in Lemma 7. Thus $\overline{\omega_{f_{0}}} \subset U_{\varepsilon}\left(\overline{\left.\omega_{f}\right)}\right.$. By $(16)$ and (15)

$$
R C\left(f_{0}\right)=\Omega\left(f_{0}\right)=\overline{\omega_{f_{0}}} \subset U_{\varepsilon}\left(\overline{\omega_{f}}\right) \subset U_{\varepsilon}(\Omega(f)) \subset U_{\varepsilon}(C R(f))
$$

which means lower semi-continuity at $f_{0}$ of all considered mappings.

Lemma 5 completes the proof.

\section{References}

[1] R. Bowen, Equilibrium states and the ergodic theory of Anosov diffeomorphisms (Lecture Notes in Math. 470 (1975)).

[2] C. Conley, Isolated invariant sets and the Morse index (Amer. Math. Soc., 1978).

[3] M. Hirsch and C. Pugh, 'Stable manifolds and hyperbolic sets', Proc. Sympos. Pure Math., Vol 14, pp. 133-164 (Amer. Math. Soc., Providence, Rhode Island, 1970).

[4] M. Hurley, 'Attractors: persistence and density of their basins', Trans. Amer. Math. Soc. 269 (1982), 247-271.

[5] M. Hurley, 'Bifurcation and chain recurrence', Ergodic Theory Dynamical Systems 3 (1983), 231-240.

[6] M. Hurley, 'Consequences of topological stability', J. Differential Equations 54 (1984), 60-72.

[7] Z. Nitecki, Differentiable dinamics (M.I.T., Cambridge, Mass., 1971).

[8] M. Shub, Stabilité globale des systèmes dynamiques, (Astérisque 56 (1978)).

[9] P. Walters, On the pseudo orbits tracing property and its relationship to stability, pp. 231-244 (Lecture Notes in Math. 668).

Instytut Matematyki

Uniwersytet Jagiellonski

ul Reymonta 4, 30059 Kraków

Poland 